\title{
Acumulaciones de detritos leñosos en un cauce de montaña de Tierra del Fuego: análisis de la movilidad y de los efectos hidromorfológicos
}

\author{
LW jams in a mountain stream of Tierra del Fuego: piece movement and \\ hydro-morphological effects analysis
}

\author{
Luca Mao $^{\mathrm{a}, b^{*}}$, Sarah Burns ${ }^{\mathrm{c}}$, Francesco Comitib ${ }^{\mathrm{b}}$, Andrea Andreoli ${ }^{\mathrm{b}, \mathrm{d}}$, Adriana Urciuolo ${ }^{\mathrm{e}}$, \\ Marcelo Gaviño-Novilloc, Rodolfo Iturraspe ${ }^{\mathrm{e}}$, Mario Aristide Lenzi ${ }^{\mathrm{b}}$ \\ *Autor de correspondencia: ${ }^{a}$ University of Hull, Department of Geography, UK, L.Mao@hull.ac.uk \\ bUniversità di Padova, Dipartimento Territorio e Sistemi Agro-Forestali, Italia. \\ cUniversidad Nacional de La Plata, Departamento de Hidráulica, La Plata, Argentina. \\ dUniversidad de Concepción, Departamento de Manejo de Bosque y Medio Ambiente, Concepción, Chile. \\ eSubsecretaría de Recursos Naturales de Tierra del Fuego, Ushuaia, Argentina.
}

\begin{abstract}
SUMMARY
This work reports on the geomorphic role of large wood pieces and jams in a third order mountain stream located in Southern Tierra del Fuego (Argentina), and draining an old-growth nothofagus forested basin not influenced by beaver damming activity. Even if the in-stream number of wood pieces $(2,300)$ is comparable to that observed in other climatic areas, the slow growth of the nothofagus forest causes a lower wood abundance in terms of volumetric load $\left(121 \mathrm{~m}^{3} \mathrm{ha}^{-1}\right)$. Due to the relatively small dimensions of the large wood pieces located inside bankfull edges ( $83 \%$ of the total surveyed pieces), almost the $70 \%$ of them demonstrated to have been transported by runoff and $6 \%$ derived from bank erosion or landslides. Wood jams exert a significant influence on the channel morphology, being responsible for the creation of $30 \%$ of pools. The geomorphic influence of LW jams is also exerted by a considerable sediment storing capacity (about $1,750 \mathrm{~m}^{3}$ ). The LW-forced pool volume is strongly and positively correlated to the height of the LW jam. The results confirm that dead wood pieces, especially when organized in jams, play an important geomorphic role also in sub-Antarctic streams. The amount of large wood pieces quantified in the study site represents reference values for the assessment of the geomorphic effects of beaver activity in other Tierra del Fuego streams.
\end{abstract}

Key words: large woody debris, channel morphology, logs transport, flow resistance, sediment retention.

\section{RESUMEN}

Se analizaron los volúmenes de material leñoso y las tipologías de acumulaciones observadas en el cauce del torrente Buena Esperanza, uno de los pocos cursos de agua de Tierra del Fuego (Argentina) no afectado por la presencia de castores. Aunque el número de piezas leñosas medidas en el cauce (2.300 elementos) fue comparable a lo reportado en otras áreas geográfica-climáticas, se estimó una menor abundancia en términos de volumen total de detritos leñosos $\left(121 \mathrm{~m}^{3} \mathrm{ha}^{-1}\right)$ debido al lento crecimiento del bosque de Nothofagus spp. en estas latitudes. El $83 \%$ de los detritos leñosos se localizó dentro del canal activo, del cual el $75 \%$ presentó señales de haber sido transportado por la corriente, el 6\% se asoció a aportes laterales al torrente por deslizamientos y erosión de márgenes, y el resto a residuos de cosecha. Las dimensiones relativamente pequeñas del material leñoso se reflejaron en las tipologías de acumulaciones de troncos individualizadas, prevaleciendo las de tipo mixtas (63\%). Se estimó que las acumulaciones mixtas influyen en la morfología del cauce, siendo responsables de la retención de un volumen de $1.750 \mathrm{~m}^{3}$ de sedimentos depositados y de la creación del 30\% de las pozas. Finalmente, se identificó una correlación positiva significativa entre los volúmenes de las pozas y la altura de las acumulaciones. Los resultados destacan la importancia hidromorfológica de las acumulaciones leñosas, y representan una referencia para la evaluación de los impactos determinados por las colonias de castores en otros ríos de Tierra del Fuego.

Palabras clave: morfología de ríos, transporte de troncos, resistencia al flujo, retención de sedimentos.

\section{INTRODUCCIÓN}

Los troncos y fragmentos de plantas leñosas (LW, del anglosajón large wood $^{1}$ ) influencian fuertemente el aspecto

1 Ver glosario de términos traducidos al final del trabajo. y la funcionalidad ecológica de los cauces de los ríos de montaña debido a que aumentan la complejidad morfológica, los hábitats y la diversidad biológica (Anderson 1982). Este efecto se observa especialmente cuando los troncos se encuentran organizados en acumulaciones (Gurnell y Sweet 1998, Gurnell et al. 2002, Cordova et al. 2007). Las acumulaciones de LW afectan el comportamiento hidráulico 
y el transporte y almacenamiento de sedimentos (Keller y Swanson 1979, Gurnell y Sweet 1998), condicionando la tipología y distribución de las formas de fondo del cauce. La tipología y la forma de las acumulaciones de LW dependen de la morfología del cauce, de los procesos de reclutamiento y de las dimensiones de las piezas de detrito leñoso en el sistema fluvial (Keller y Swanson 1979, Swanson y Lienkaemper 1984, Bilby y Ward 1989). Abbe y Montgomery (2003) identificaron y describieron distintos tipos de acumulaciones de LW, caracterizando cada una de ellas en función de la estabilidad, de los efectos geomorfológicos y de la distribución a lo largo del sistema fluvial.

La presencia de LW en el cauce influye en el comportamiento hidráulico, siendo una fuente adicional de resistencia, tanto en ríos de baja pendiente (Shields y Gippel 1995) como en ríos de montaña. En torrentes con formas de fondo definidas (Lenzi 1999, Mintegui Aguirre et al. 2006) en secuencias escalón-remanso, MacFarlane y Wohl (2003) midieron mayor resistencia hidráulica debido a la presencia adicional de detrito leñoso. Wilcox et al. (2006) exploraron, a través de experimentos de laboratorio, la partición entre las componentes de resistencia hidráulica debida a las partículas del lecho y al detrito leñoso en las secuencias escalón-remanso, y revelaron cómo ambas componentes son dominantes en torrentes a elevada pendiente, aunque efectos de interacción dificulten la diferenciación entre una y otra.

El aumento de la complejidad de hábitats relacionado con la formación de pozas de socavación, aguas abajo de un salto o escalón, es uno de los efectos ecológicos de los LW en ríos de montaña. Montgomery et al. (1995) encontraron una correlación directa entre la abundancia de LW y la frecuencia de pozas, así como hay directa relación entre las dimensiones de los LW y el volumen de las pozas de socavación que se forman aguas abajo de estas estructuras (Bilby y Ward 1989, Keller y Swanson 1979). En general, la interdistancia de las pozas es función de la abundancia de LW en el cauce, de las dimensiones de LW, de la pendiente y del ancho del cauce en condiciones de caudal de cauce lleno, o sea, en condiciones de máxima descarga que el canal puede encauzar sin invadir su planicie de inundación (Bilby y Ward 1989, Montgomery et al. 1995, Mintegui Aguirre et al. 2006). No obstante, en ríos con mayores pendientes, la interdistancia entre las pozas es menos sensible a la abundancia de LW que en ríos con pendiente más moderada (Montgomery et al. 1995).

La influencia de LW en la forma y en la dinámica del cauce es mayor en ríos de primer orden o de cabecera, donde el LW presenta un rol clave en la retención de sedimentos (Nakamura y Swanson 1993, Faustini y Jones 2003). El "orden" es una manera de clasificar en orden jerárquico las dimensiones de los cauces: los ríos permanentes más pequeños son de primer orden, la confluencia de dos ríos de primer orden genera un río de segundo orden, etcétera; mientras que un río entrante en otro mayor no cambia el orden de este último (Strahler 1952).

Marston (1982) encontró que en ríos de tercer a quinto orden los sedimentos almacenados por acumulaciones de LW pueden ser mayores al $100 \%$ de la producción total anual de sedimentos. Evaluaciones similares fueron proporcionadas por Swanson y Lienkaemper (1978), y Swanson y Fredriksen (1982). La remoción de LW de los ríos de cabecera puede aumentar el transporte de sedimentos de un orden de magnitud (Smith et al. 1993), mientras que las acumulaciones de LW reducen la eficiencia del transporte de sedimentos en relación a cauces sin ellas (Swanson y Lienkaemper 1978, Thompson 1995).

Si bien existe información cuantitativa en relación a la abundancia de material leñoso en los cauces, el conocimiento científico sobre el inicio del transporte del material leñoso, su movilidad y su depósito es, hasta el momento, sólo de tipo pionero. Estudios previos indican que los troncos pequeños tienen mejor movilidad que los grandes (Lienkaemper y Swanson 1987, Young 1994), que la frecuencia de movimiento de los troncos crece cuando aumenta la dimensión del curso de agua (Lienkaemper y Swanson 1987, Bilby y Ward 1989) y que los elementos más móviles son los que tienen una longitud menor a la anchura del río en condiciones de cauce lleno (Nakamura y Swanson 1993). Abbe y Montgomery (2003) sugieren que los troncos tienden a ser estables si su longitud es mayor de 1,5 veces la anchura del río en condiciones de cauce lleno. Para pequeños cauces de montaña, Lienkaemper y Swanson (1987) decrecen este límite a la igualdad entre longitud del tronco y anchura de cauce lleno. No obstante algunos ensayos de laboratorio (Ishikawa 1989, Braudick y Grant 2000), no hay hoy en día a disposición modelos suficientemente calibrados de movilidad de detritos leñosos en los cauces.

La mayor parte de las investigaciones relacionadas al material leñoso en ríos ha sido realizada en el noroeste de Norte América, en Europa y en Oceanía. Recientemente se presentó una primera descripción cuantitativa de la abundancia y características de los LW en ríos de montaña en el sur de Los Andes (Comiti et al. 2008, Andreoli 2007, Andreoli et al. 2007). El presente trabajo se centra en el rol hidro-geomorfológico de los elementos de LW y de las acumulaciones en un río de montaña de tercer orden localizado en el sur de Tierra del Fuego (Argentina). El río en estudio drena una cuenca con bosques nativos de Nothofagus spp. y es uno de los pocos cauces en Tierra del Fuego que no está afectado por la presencia del castor (Castor canadensis Kuhl). Desde su introducción en 1946, el castor se extendió en todo el archipiélago fueguino, demostrando alta capacidad invasora (Lizarralde et al. 2004). La consecuencia de su actividad es el consistente aporte de material leñoso al sistema fluvial y la inundación del bosque de ribera.

Este trabajo tiene como objetivo reportar los resultados de un estudio tendiente a determinar la cantidad de detrito, 
las tipologías y dimensiones de las acumulaciones leñosas, y las consecuentes modificaciones de la morfología fluvial en un torrente de Tierra del Fuego (Argentina) debido a las características de las mismas acumulaciones leñosas dentro del cauce, y comparar los resultados con cuencas situadas en otras regiones del mundo. El estudio describe el impacto y los efectos de acumulaciones de LW, tanto en la interdistancia que se establece entre las pozas de socavación como en la resistencia al flujo y en la retención del sedimento, y proporciona además informaciones cuantitativas sobre la movilidad y el transporte de troncos en ocasión de una crecida con caudal pico cercano al valor de cauce lleno.

\section{MÉTODOS}

Área de estudio. El estudio se llevó a cabo en la cuenca del torrente Buena Esperanza $\left(12,9 \mathrm{~km}^{2}\right)$, ubicada en la región argentina de Tierra del Fuego, el cual fluye al Canal de Beagle atravesando la ciudad de Ushuaia. La región se identifica climáticamente como templada fría húmeda y el régimen hidrológico de la cuenca es glacionival. Los rangos de elevación de la cuenca van desde 0 a 1.275 m s.n.m. La porción de la cuenca (34\%) debajo del límite de vegetación (550-650 m s.n.m.) está cubierta de bosque mixto magallánico (Nothofagus pumilio Krasser en asociación con $N$. antarctica Forster y $N$. betuloides Oerst) (Barrera et al. 2000). Las condiciones climáticas extremas que llevan a un crecimiento lento de Nothofagus spp. (Rebertus et al. 1997, Barrera et al. 2000) producen una cobertura forestal madura con árboles que presentan diámetros a la altura del pecho generalmente inferiores a 0,5 m. Solamente pequeñas áreas boscosas fueron aprovechadas durante la década del '40. El torrente Buena Esperanza es uno de los pocos cauces no impactados por la presencia del castor en Tierra del Fuego (Lizarralde et al. 2004), posiblemente debido a la cercanía de la ciudad de Ushuaia, y de los caminos que pasan cerca del cauce en la parte baja de la cuenca.

Levantamientos topográficos. En febrero - marzo de 2006 se realizaron mediciones de campo en un tramo de $1,85 \mathrm{~km}$ del cauce principal del torrente Buena Esperanza ubicado dentro de la porción boscosa de la cuenca. La sección final aguas abajo corresponde a un vertedero que provee de agua potable a la ciudad de Ushuaia con registros de caudales desde el año 2000. El perfil longitudinal del cauce se obtuvo mediante el uso de un distanciómetro láser con clinómetro. En total, se identificaron 33 tramos individuales dependiendo de la uniformidad de pendiente, ancho del cauce y abundancia de material leñoso (cuadro 1). Las variables del cauce medidas en el campo en cada tramo incluyen el ancho medio del río en condiciones de cauce lleno $\left(B_{B F}\right)$, el ancho medio de la planicie de inundación $\left(B_{F P}\right)$, la profundidad media en condiciones de cauce lleno $\left(H_{B F}\right)$, el número de escalones-saltos más altos de $H_{B F}$ y el número de rocas (peñones) con diámetro mayor de $H_{B F}$. El tirante correspondiente al valor de caudal de cauce lleno se estimó en campo considerando la altura del límite más bajo de vegetación, cambios en el tamaño del sedimento en las barras laterales, y residuo orgánico depositado por el flujo (Montgomery y Buffington 1997, Mintegui Aguirre et al. 2006). La profundidad (tirante) y el ancho correspondientes, ya sea al nivel de caudal de cauce lleno como al de la planicie de inundación (área de inundación), se determinaron utilizando los levantamientos topográficos realizados en tres secciones transversales emplazadas en cada tramo del cauce. Los perfiles longitudinales determinados a partir de los levantamientos topográficos en campo también se utilizaron para derivar la geometría de las formas morfológicas de escalones-saltos (Lenzi 1999), tanto para los escalones constituidos por bloques o peñones, como para los constituidos por acumulaciones de troncos y detritos leñosos.

Medición de los elementos y de las acumulaciones leñosas. Todos los troncos y los trozos de LW mayores a $10 \mathrm{~cm}$ de diámetro y $1 \mathrm{~m}$ de largo (Abbe y Montgomery 2003, Faustini y Jones 2003, Comiti et al. 2006, 2008, Andreoli et al. 2007) fueron medidos con cinta y forcípula tanto en el cauce activo como en la planicie de inundación adyacente. El volumen de cada elemento se calculó a partir de su diámetro medio y de su largo, asumiendo una forma de cilindro sólido (Cordova et al. 2007).

Además de los elementos leñosos singulares, se midieron todas las acumulaciones leñosas, que fueron también clasificadas según Abbe y Montgomery (2003) en tres tipos, dependiendo del origen de sus elementos constituyentes. Dichas acumulaciones fueron: a) autóctonas: elementos clave no transportados fluvialmente, por lo tanto provenientes de los márgenes o de la planicie de inundación del cauce en las proximidades cercanas a la ubicación de la acumulación; b) de transporte: elementos clave transportados desde tramos de aguas arriba, y c) acumulaciones mixtas: elementos clave autóctonos que capturaron troncos transportados desde aguas arriba. Aunque la metodología de Abbe y Montgomery (2003) es más compleja e implica un segundo nivel de clasificación, en este sitio de estudio por simplicidad se identificaron las acumulaciones autóctonas con los escalones de troncos (elementos clave caídos transversalmente en el cauce desde los márgenes y formando un escalón), no se distinguieron las acumulaciones de transporte en subcategorías (así como en la clasificación original de Abbe y Montgomery (2003)) y se diferenciaron las acumulaciones mixtas en diques leñosos (acumulaciones de ancho igual o mayor al ancho del cauce), acumulaciones a espigón (de ancho inferior al del cauce) y acumulaciones combinadas (claramente compuestas por elementos clave autóctonos y otros troncos procedentes desde aguas arriba, pero con características intermedias entre los diques leñosos y las acumulaciones 
Cuadro 1. Características morfológicas, dimensionales y de presencia de detrito leñoso en los tramos medidos en el torrente Buena Esperanza. $L$ es la longitud del tramo, $i$ es su pendiente, $B_{B F}$ y $H_{B F}$ son el ancho medio y el tirante medio en condiciones de caudal de cauce lleno. El tramo 8 no fue medido debido a su inaccesibilidad.

Main characteristics of surveyed reaches. $L$ is channel length, $i$ is reach slopes, $B_{B F}$ is mean bankfull width and $H_{B F}$ is mean bankfull height. Reach 8 were not surveyed because of inaccessibility.

\begin{tabular}{|c|c|c|c|c|c|c|c|}
\hline Tramo & Morfología dominante & $\begin{array}{c}L \\
(\mathrm{~m})\end{array}$ & $\begin{array}{c}i \\
\left(\mathrm{~m} \mathrm{~m}^{-1}\right)\end{array}$ & $\begin{array}{l}\mathrm{B}_{\mathrm{BF}} \\
(\mathrm{m})\end{array}$ & $\begin{array}{r}\mathrm{H}_{\mathrm{BF}} \\
(\mathrm{m})\end{array}$ & $\begin{array}{c}\text { Densidad espacial } \\
\text { de LW } \\
\left(\text { piezas } \mathrm{ha}^{-1}\right) \\
\end{array}$ & $\begin{array}{c}\text { Densidad lineal } \\
\text { de LW } \\
(\text { piezas km-1) }\end{array}$ \\
\hline 1 & Rápidos & 62,3 & 0,02 & 10,5 & 0,47 & 429 & 450 \\
\hline 2 & Rápidos & 47,2 & 0,04 & 6,6 & 0,42 & 831 & 551 \\
\hline 3 & Rápidos & 37,0 & 0,06 & 7,0 & 0,51 & 581 & 405 \\
\hline 4 & Acumulaciones de detrito leñoso & 44,7 & 0,06 & 8,7 & 0,53 & 1.781 & 1.543 \\
\hline 5 & Secuencias escalón-remanso & 34,8 & 0,09 & 6,0 & 0,63 & 904 & 545 \\
\hline 6 & Secuencias escalón & 48,9 & 0,05 & 9,1 & 0,60 & 2.292 & 2.085 \\
\hline 7 & Secuencias escalón & 71,9 & 0,08 & 6,3 & 0,65 & 1.944 & 1.225 \\
\hline 8 & Cañón & 230,0 & - & - & - & - & - \\
\hline 9 & Acumulaciones de detrito leñoso & 70,4 & 0,06 & 4,1 & 0,45 & 4.229 & 1.748 \\
\hline 10 & Secuencias escalón-remanso & 40,8 & 0,04 & 3,8 & 0,42 & 1.919 & 736 \\
\hline 11 & Acumulaciones de detrito leñoso & 82,3 & 0,07 & 6,6 & 0,39 & 2.706 & 1.786 \\
\hline 12 & Acumulaciones de detrito leñoso & 52,5 & 0,06 & 4,9 & 0,39 & 2.914 & 1.428 \\
\hline 13 & Acumulaciones de detrito leñoso & 71,1 & 0,07 & 7,7 & 0,57 & 2.146 & 1.661 \\
\hline 14 & Secuencias escalón-remanso & 102,7 & 0,07 & 7,5 & 0,31 & 717 & 535 \\
\hline 15 & Secuencias escalón-remanso & 60,0 & 0,05 & 4,7 & 0,45 & 850 & 400 \\
\hline 16 & Rápidos & 44,1 & 0,06 & 4,2 & 0,37 & 756 & 317 \\
\hline 17 & Secuencias escalón-remanso & 46,1 & 0,06 & 5,1 & 0,38 & 1.156 & 586 \\
\hline 18 & Acumulaciones de detrito leñoso & 59,3 & 0,08 & 5,5 & 0,37 & 1.128 & 624 \\
\hline 19 & Acumulaciones de detrito leñoso & 70,8 & 0,08 & 5,4 & 0,40 & 2.001 & 1.074 \\
\hline 20 & Secuencias escalón-remanso & 49,3 & 0,08 & 5,9 & 0,47 & 2.015 & 1.196 \\
\hline 21 & Acumulaciones de detrito leñoso & 83,2 & 0,07 & 5,8 & 0,40 & 2.460 & 1.419 \\
\hline 22 & Acumulaciones de detrito leñoso & 59,4 & 0,07 & 5,2 & 0,43 & 2.765 & 1.447 \\
\hline 23 & Escalón de troncos & 69,4 & 0,08 & 8,3 & 0,44 & 1.865 & 1.541 \\
\hline 24 & Acumulaciones de detrito leñoso & 61,4 & 0,06 & 7,0 & 0,39 & 2.059 & 1.434 \\
\hline 25 & Rápidos & 81,4 & 0,06 & 4,7 & 0,37 & 1.264 & 590 \\
\hline 26 & Rápidos & 68,9 & 0,06 & 5,2 & 0,33 & 870 & 450 \\
\hline 27 & Rápidos & 40,9 & 0,05 & 4,6 & 0,42 & 372 & 171 \\
\hline 28 & Rápidos & 58,4 & 0,08 & 4,9 & 0,40 & 1.434 & 703 \\
\hline 29 & Secuencias escalón-remanso & 41,3 & 0,07 & 5,2 & 0,41 & 845 & 436 \\
\hline 30 & Acumulaciones de detrito leñoso & 39,3 & 0,07 & 5,6 & 0,36 & 3.384 & 1.906 \\
\hline 31 & Acumulaciones de detrito leñoso & 47,4 & 0,07 & 6,1 & 0,38 & 3.581 & 2.196 \\
\hline 32 & Secuencias escalón-remanso & 57,2 & 0,08 & 5,8 & 0,43 & 1.498 & 874 \\
\hline 33 & Secuencias escalón-remanso & 40,5 & 0,09 & 4,9 & 0,37 & 2.932 & 1.432 \\
\hline
\end{tabular}

a espigón). La terminología anglosajona se utilizó para evitar posibles equivocaciones en futuras comparaciones con estudios paralelos.

Durante la campaña de toma de datos de campo, además de la clasificación de cada una de las acumulaciones identificadas, se midieron sus dimensiones geométricas (altura $H_{J}$, ancho $B_{J}$, y largo $L_{J}$ ) asumiendo una forma de paralelepípedo sólido (Thevenet et al. 1998). El volumen de cada acumulación leñosa se estimó a partir de sus dimensiones geométricas $\left(V_{J}=H_{J}^{*} B_{J}^{*} L_{J}\right)$ sin considerar su porosidad. Para las acumulaciones que formaban un escalón en el perfil se midió también la profundidad de las pozas de socavación aguas abajo del escalón $(s)$ y su longitud $(L p)$. El volumen de sedimentos acumulados aguas arriba de las acumulaciones fue estimado como un paralelepípedo sólido sin considerar la porosidad del sedimento. Además, se tomó nota de las dimensiones y origen de los elementos clave, e información cualitativa de los efectos 
morfológicos de cada acumulación (protección o erosión de las márgenes del cauce, desviación o bifurcación del cauce, retención de sedimentos, etc.).

Medición de la distancia de transporte de los elementos leñosos. Con la finalidad de cuantificar la distancia de transporte de los troncos frente a una crecida, durante la campaña de toma de datos de campo de febrero y marzo de 2006 fueron marcados 380 troncos con etiquetas metálicas numeradas. En la elección de los elementos leñosos a marcar se seleccionaron troncos con diámetro entre 0,1 y $0,95 \mathrm{~m}$ y con longitud entre 0,5 y $12 \mathrm{~m}$. La longitud y el diámetro de todos los elementos fueron medidos con una cinta métrica. Además, se registraron informaciones cualitativas acerca de la posición (dentro o afuera del nivel de cauce lleno), la orientación (paralelo, ortogonal o transversal a la corriente), el grado de libertad (libre o vinculado) y la organización (aislado o en acumulación) del tronco en el cauce. Los elementos de dimensión mayor fueron marcados con más de una etiqueta, con el objetivo de seguir los movimientos de los pedazos que pudieran generarse de la rotura de algún elemento de LW. La posición de los troncos marcados fue medida en campo y registrada, como distancia absoluta y relativa entre los elementos y los 34 piquetes posicionados a lo largo del cauce.

Medición de la resistencia al flujo. Con el fin de cuantificar la influencia del detrito leñoso en la resistencia al flujo, varias mediciones de velocidad media del flujo fueron realizadas en tramos caracterizados por su presencia o ausencia (cuadro 2). La misma metodología fue aplicada en el torrente Tres Arroyos, situado en la Reserva Nacional Malacahuello-Nalcas (IX Región de Chile), donde se llevó a cabo una larga campaña de medición del transporte sólido (Uyttendaele 2006, Mao et al. 2008) y de detrito leñoso en el cauce (Comiti et al. 2007, Andreoli 2007, Andreoli et al. 2007). La velocidad media del flujo en un tramo fue medida en campo usando un trazador salino $(\mathrm{NaCl})$. Dos conductivímetros (Campbell CR510 con sonda CS547) fueron posicionados en los extremos superior e inferior de cada tramo. El trazador salino fue introducido instantáneamente en el flujo a una distancia suficientemente aguas arriba (10 veces la anchura del canal) del extremo superior del tramo para permitir una adecuada mezcla lateral de la solución salina (Elder et al. 1990). La mezcla salina introducida en el cauce aumentaba la conductividad eléctrica y, en las cantidades empleadas, no proporcionaba ningún impacto a la fauna íctica y a la población de invertebrados bentónicos (Wood y Dykes 2002). El intervalo temporal registrado entre la llegada del pico de conductividad eléctrica en los dos conductivímetros, junto con la distancia entre estos dos puntos (medida con un distanciómetro láser), fueron utilizados para calcular la velocidad media del flujo en cada tramo. La resistencia al flujo, expresada como parámetro de Darcy-Weisbach (f), fue calculada a partir de la velocidad media del flujo $(V)$ a través de

$$
f=\frac{8 g h i}{V^{2}}
$$

donde $g$ es la aceleración de la gravedad $\left(9,8 \mathrm{~m} \mathrm{~s}^{-1}\right), h$ es el tirante medio de la corriente calculado usando la ecuación de continuidad y el ancho de la sección bañada media medida en campo, $i$ es la pendiente del tramo $\left(\mathrm{m} \mathrm{m}^{-1}\right)$. El método del trazante salino para la evaluación de la resistencia al flujo en ríos de alta pendiente y con flujo muy turbulento ha sido probado y empleado con éxito en estudios de campo anteriores (Lee y Ferguson 2002, MacFarlane y Wohl 2003, Curran y Wohl 2003, Wohl y Wilcox 2005, Comiti et al. 2007). La altura media $(H)$ y la longitud media $(L)$ de las formas morfológica de escalónremanso fueron calculadas a partir del perfil longitudinal de cada tramo medido en campo (cuadro 2). La granulometría superficial representativa de cada tramo (cuadro 2) fue medida con un muestreo de grilla (grid-by-number) de por lo menos 100 gránulos para cada muestra.

\section{RESULTADOS}

Abundancia de troncos y características de las acumulaciones. Se midieron más de 2.300 piezas de detrito leñoso (LW) a lo largo de los 33 tramos de canal observados. El $83 \%$ de ellas fueron localizadas dentro del canal activo (anchura correspondiente al valor de caudal de cauce lleno). El 74,8\% de las piezas de LW en el canal activo presentaba señales de haber sido transportado por la corriente desde aguas arriba al tramo donde fueron observadas, mientras que el 5,7\% llegó al tramo de cauce analizado desde laderas erosionadas o deslizamientos. Los residuos de cosecha sumaban un 2,5\% del total de las piezas medidas en el canal activo.

El número de piezas leñosas por tramo expresadas como densidad areal y lineal se reportan en el cuadro 1. La densidad espacial volumétrica promedio calculada fue de $121 \mathrm{~m}^{3} \mathrm{ha}^{-1}\left(76 \mathrm{~m}^{3} \mathrm{~km}^{-1}\right)$, pero se evidenció una gran variabilidad entre tramos, desde 20 hasta $395 \mathrm{~m}^{3}$ ha $^{-1}$. La densidad espacial de LW varió entre 372 y 4.229 piezas $\mathrm{ha}^{-1}$, con un valor promedio de 1.770 piezas ha ${ }^{-1}$ (1.047 piezas $\left.\mathrm{km}^{-1}\right)$.

En total, 113 acumulaciones de LW fueron identificadas, clasificadas según Abbe y Montgomery (2003) y medidas en sus dimensiones en el torrente Buena Esperanza (cuadro 3). El 27\% de las acumulaciones fueron escalones (saltos) de troncos, completos o parciales (figura 1), siendo el único tipo de acumulación autóctona, ya que los aportes de acumulaciones de las márgenes del cauce fueron siempre asociados a material leñoso flotante atrapado, creando acumulaciones mixtas. Treinta y tres de las acumulaciones de LW (28\%, figura 1) fueron clasificadas como acumulaciones combinadas por estar claramente compuestas por elementos clave autóctonos y otros troncos 
Cuadro 2. Características morfológicas y dimensionales de los tramos (torrentes Buena Esperanza, Argentina, y Tres Arroyos, Chile) donde se ha medido la resistencia hidráulica. En la tercera columna, $X$ indica los tramos dominados morfológicamente por la presencia de acumulaciones de LW, $L$ indica la longitud del tramo, $i$ su pendiente, $D_{84}$ el percentil 84 de la curva granulométrica acumulada, $H s$ es la altura media de los escalones y $L s$ es la interdistancia media entre los escalones.

Characteristics of channel reaches and units considered for flow resistance in Buena Esperanza and Tres Arroyos. $X$ identifies the reaches where the LW jams dominate the channel morphology. $L$ is reach length, $i$ is slope, $D_{84}$ is the $84^{\text {th }}$ percentile of grain size distribution and $H s$ and $L s$ indicate mean step height and step length, respectively.

\begin{tabular}{|c|c|c|c|c|c|c|c|c|}
\hline & Tramo & LW & Morfología & $\begin{array}{c}L \\
(\mathrm{~m})\end{array}$ & $\begin{array}{c}i \\
\left(\mathrm{~m} \mathrm{~m}^{-1}\right)\end{array}$ & $\begin{array}{l}D_{84} \\
(\mathrm{~m})\end{array}$ & $\begin{array}{l}H s \\
(\mathrm{~m})\end{array}$ & $\begin{array}{c}L s \\
(\mathrm{~m})\end{array}$ \\
\hline \multirow{10}{*}{ 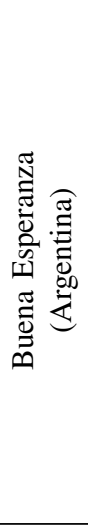 } & A & - & Secuencias escalón-remanso & 21,5 & 0,022 & 0,223 & 0,25 & 2,4 \\
\hline & $\mathrm{C}$ & - & Secuencias escalón-remanso & 17,2 & 0,023 & 0,113 & 0,22 & 1,9 \\
\hline & E & - & Rápidos & 23,4 & 0,076 & 0,260 & 0,42 & 6,0 \\
\hline & G & - & Secuencias escalón-remanso & 17,0 & 0,103 & 0,336 & 0,61 & 3,4 \\
\hline & B & $\mathrm{X}$ & Dos grandes diques leñosos & 22,8 & 0,097 & 0,083 & 0,99 & 3,6 \\
\hline & $\mathrm{D}$ & $\mathrm{X}$ & Escalón de troncos & 4,1 & 0,105 & 0,172 & 1,00 & 2,7 \\
\hline & $\mathrm{F}$ & $\mathrm{X}$ & Diques leñosos & 13,7 & 0,092 & 0,208 & 1,02 & 2,3 \\
\hline & DEF & $\mathrm{X}$ & Diques leñosos & 41,3 & 0,084 & 0,219 & 0,72 & 4,3 \\
\hline & $\mathrm{GH}$ & $\mathrm{X}$ & Diques leñosos & 22,6 & 0,132 & 0,285 & 0,78 & 3,9 \\
\hline & $\mathrm{H}$ & $\mathrm{X}$ & Diques leñosos & 5,6 & 0,223 & 0,153 & 1,28 & 5,3 \\
\hline \multirow{5}{*}{ 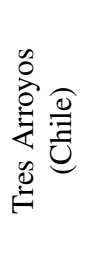 } & 15 & - & Secuencias escalón-remanso & 36,9 & 0,028 & 0,151 & 0,37 & 13,7 \\
\hline & 1 & - & Secuencias escalón-remanso & 23,9 & 0,057 & 0,378 & 0,21 & 9,7 \\
\hline & 14 & - & Secuencias escalón-remanso & 50,5 & 0,072 & 0,341 & 0,53 & 4,8 \\
\hline & 8 & - & Secuencias escalón-remanso & 42,1 & 0,036 & 0,385 & 0,63 & 5,8 \\
\hline & 10 & $\mathrm{X}$ & Escalón de troncos & 32,1 & 0,153 & 0,143 & 1,89 & 10,6 \\
\hline
\end{tabular}

Cuadro 3. Caracterización de las acumulaciones de LW en el torrente Buena Esperanza (A: acumulaciones autóctonas; M: acumulaciones mixtas; $\mathrm{T}$ : acumulaciones de transporte).

Characterization of surveyed LW jams (A: autochthonous jams; C: combination jams; T: transport jams).

\begin{tabular}{lcccc}
\hline $\begin{array}{l}\text { Acumulaciones } \\
\text { leñosas }\end{array}$ & Tipo & Número & $\begin{array}{c}\text { Número } \\
\text { por km }\end{array}$ & $\begin{array}{c}\text { Número } \\
\text { medio de } \\
\text { elementos } \\
\text { de LW }\end{array}$ \\
\hline $\begin{array}{l}\text { Escalón } \\
\text { de troncos }\end{array}$ & $\mathrm{A}$ & 30 & 16,2 & 4,3 \\
$\begin{array}{l}\text { Acumulaciones } \\
\text { combinadas }\end{array}$ & $\mathrm{M}$ & 33 & 17,8 & 7,8 \\
$\begin{array}{l}\text { Acumulaciones } \\
\text { a espigón }\end{array}$ & $\mathrm{M}$ & 11 & 5,9 & 10,1 \\
$\begin{array}{l}\text { Diques } \\
\text { leñosos }\end{array}$ & $\mathrm{M}$ & 27 & 6,5 & 13,8 \\
$\begin{array}{l}\text { Acumulaciones } \\
\text { alóctonas }\end{array}$ & $\mathrm{T}$ & 12 & 2,2 & 5,0 \\
\hline
\end{tabular}

procedentes desde aguas arriba (acumulaciones mixtas); es decir, por poseer características que excluyen las de dique leñoso o las de acumulación a espigón. En total, las acumulaciones mixtas (diques leñosos, acumulaciones a espigón y combinadas) representaron la tipología dominante (62\%, figura 1). Acumulaciones de transporte, o sea totalmente constituidas por elementos transportados desde aguas arriba, representaron sólo el 10\% del total de las acumulaciones de LW observadas en el torrente Buena Esperanza (cuadro 3).

Influencia de las acumulaciones de LW en la morfología del cauce. Se reconocieron varios efectos de las acumulaciones leñosas en la morfología del cauce del torrente Buena Esperanza. Se pudieron observar nueve principales tipologías de consecuencias morfológicas (figura 2), muchas veces manifestadas de forma múltiple. Como era de esperar, todos los escalones de troncos causaron una socavación aguas abajo, pero sólo el $50 \%$ de ellos determinó una acumulación detectable de sedimentos aguas arriba. Estos dos efectos geomorfológicos caracterizaron, respectivamente, más del $85 \%$ y $96 \%$ de los diques leñosos. Esto es predecible ya que los escalones de troncos y diques 


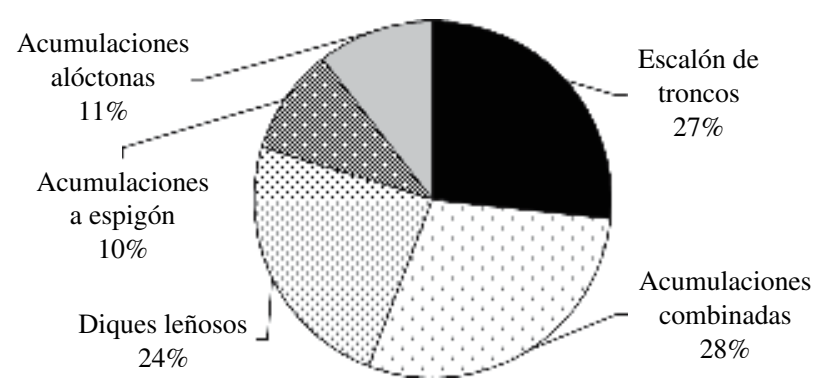

Figura 1. Tipologías de acumulaciones de LW identificadas en el cauce principal del torrente Buena Esperanza, Tierra del Fuego, Argentina.

Type of LW jams surveyed in Buena Esperanza main channel.

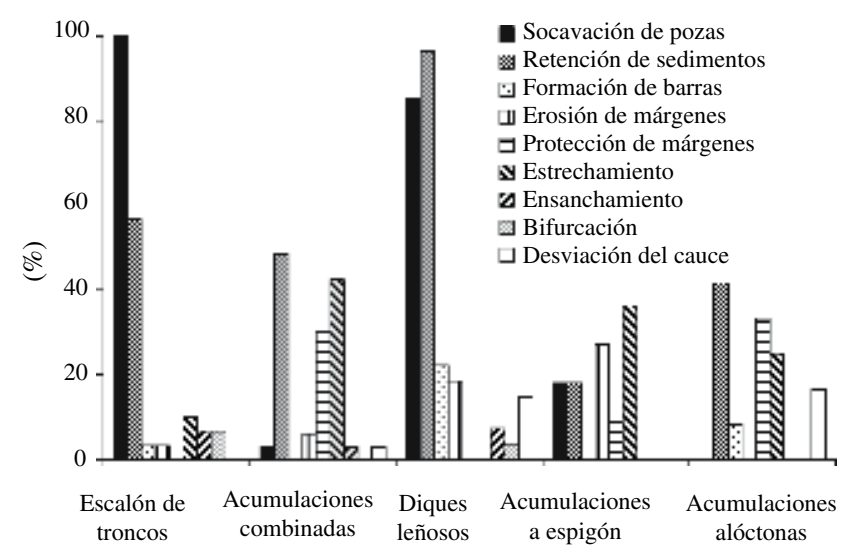

Figura 2. Influencias morfológicas de las acumulaciones de LW en el cauce del torrente Buena Esperanza.

channel.

Morphological influences of LW jams in Buena Esperanza

leñosos, que son las acumulaciones de LW que ocupan el cauce en todo su ancho, son particularmente estables e imponen el flujo a un salto que provoca un foso de socavación aguas abajo. Los diques leñosos también mostraron otros efectos secundarios ya observados por otros autores (Abbe y Montgomery 2003), tales como formaciones de barras de sedimentación aguas arriba (22\%) y erosión de las márgenes aguas abajo (18\%). Las acumulaciones a espigón, que no ocupan completamente la anchura del cauce, manifestaron sólo en el $20 \%$ de los casos una socavación aguas abajo o retención de sedimento aguas arriba. Sin embargo, produjeron estrechamientos evidentes de la sección (36\%) y la consecuente erosión de las márgenes (27\%, figura 2). En total, las acumulaciones de transporte, que en general yacen a lo largo de las márgenes, así como el estrechamiento de la sección, brindan protección directa a dichas márgenes.

La retención de sedimento también se reconoció para el $42 \%$ de las acumulaciones de transporte. Otros efectos morfológicos como formación de barras de se- dimentación, ensanchamiento, bifurcación y desviación del cauce -generalmente citado para ríos más anchos y de menor gradiente (Abbe y Montgomery 2003)- están subrepresentados (figura 2) en el cauce del torrente Buena Esperanza, probablemente debido a la fuerte pendiente y al alto confinamiento lateral del cauce.

En la campaña de toma de datos se midió la altura de los escalones $(H s)$, junto con la profundidad $(s)$ y la longitud $(L p)$ de las pozas creadas por socavación aguas abajo de las estructuras de detrito leñoso (30 escalones de troncos y 22 diques leñosos), y de los escalones de bloques (123 en total), constituidos por la organización de grandes piedras (bloques o peñones), emplazados en sentido transversal a la corriente (Chin 1989). Como se esperaba, se observó que la altura $(H s)$ de los diques leñosos fue mayor a la de los escalones de troncos y de bloques (figura 3). Aparte de un escalón de roca muy alto $(1,2 \mathrm{~m})$ en el tramo 5 , las alturas de los escalones de bloques variaron entre $0,1 \mathrm{~m} \mathrm{y}$ $0,8 \mathrm{~m}$, con un valor medio de $0,35 \mathrm{~m}$. La altura promedio de los escalones de troncos y de los diques leñosos fue casi dos y tres veces mayor, respectivamente. Considerando la profundidad media de las pozas se observó un patrón similar, pero con menores diferencias entre los escalones de troncos y los diques leñosos (figura 3). Los tres tipos de escalones fueron significativamente diferentes (prueba t, $P<0,05)$ tanto para sus alturas como para la profundidad de las pozas de socavación. Previsiblemente, $H s$ y $s$ están positivamente correlacionados $(r=0,74 ; P<0,001)$.

Comparando la altura de los escalones de troncos con el diámetro medio de los troncos que los constituyen, se observó una correlación positiva $(r=0,62 ; P<0,001)$, confirmando los resultados reportados por Wohl et al. (1997) en los escalones de troncos de los ríos del noroeste de Montana (USA). El promedio de los diámetros medios de los troncos que forman los escalones de troncos fue de $0,27 \mathrm{~m}$, comparable con el percentil 84 de los diámetros de las piezas de $\mathrm{LW}$ medidas $(0,25 \mathrm{~m})$.

Efecto de las acumulaciones de $L W$ en la resistencia al flujo. El cuadro 2 pone de manifiesto que la granulometría superficial de los tramos donde el detrito vegetal no fue dominante en la definición de la morfología del lecho $\left(0,113 \mathrm{~m}<D_{84}<0,385 \mathrm{~m}\right)$ fue mayor respecto a tramos donde el LW se presentó organizado en acumulaciones $\left(0,083 \mathrm{~m}<D_{84}<0,285 \mathrm{~m}\right)$. Esta diferencia fue estadísticamente significativa $(P<0,05)$ así como la diferencia en la relación entre altura e interdistancia de los escalones $(H s / L s ; P=0,001)$. Esta última variable indica que en tramos dominados morfológicamente por la presencia de acumulaciones de LW, los escalones fueron más altos y las pozas de socavación aguas abajo fueron más pronunciadas. Esto provocó una mayor macrorrugosidad de los tramos caracterizados por presencia de LW. En la figura 4 se ilustra la variación del índice de resistencia de Darcy-Weisbach (calculado con la ecuación 1) al variar el caudal líquido unitario adimensional $\left(q^{*}=q /\left(g D_{84}\right)^{3}\right)^{0,5}$, calculado como 


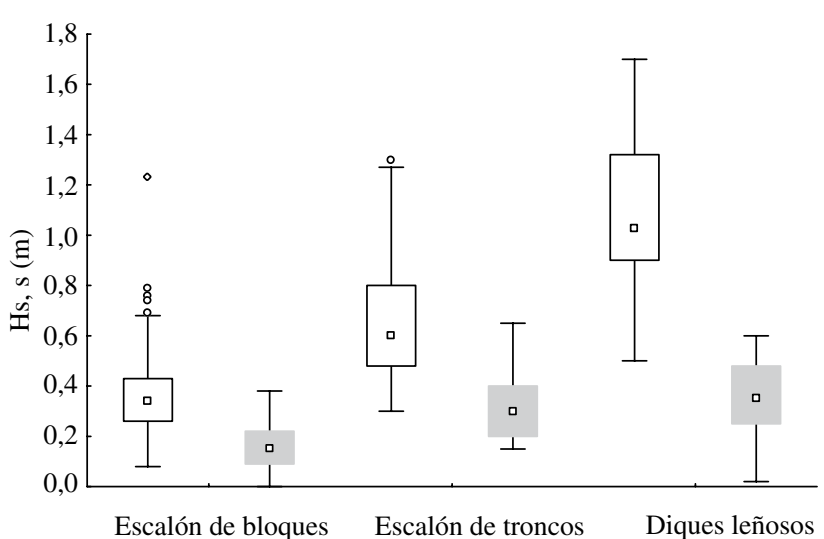

Figura 3. Comparación de la altura de los escalones (Hs, caja blanca) y la profundidad de las pozas o fosos de socavación ( $s$, caja gris), diferenciando los escalones constituidos por bloques $\mathrm{o}$ peñones, escalones de troncos y acumulaciones tipo diques leñosos. En cada diagrama la caja sólida indica el rango entre los percentiles 25 y 75, los cuadrados indican la mediana, los bigotes indican los valores máximos y mínimos no atípicos, los círculos sólidos indican los valores atípicos y los diamantes indican valores extremos.

Step height $\left(H_{s}\right.$, empty boxes) and scour depth $(s$, shaded boxes) comparison between steps created by boulders, log and valley jams. For each box plot, the solid box indicates the range between the $25^{\text {th }}$ and $75^{\text {th }}$ percentiles, the square icon indicates the median, whiskers indicate the non outlier maximum and minimum values, solid circles indicate outliers and the diamond indicates extreme value.

en Comiti et al. (2007) para los tramos considerados en el análisis (cuadro 2). El rango de $q^{*}$ explorado varió de 0,036 a 1,126, y los correspondientes valores de índice de resistencia $(f)$ variaron entre 0,3 y 97,5 . No obstante la dispersión de los valores, es evidente que al aumento del caudal correspondió una disminución del índice de resistencia; pero más notablemente se advierte que para un cierto $q^{*}$, los tramos con acumulaciones de LW proporcionaron una resistencia al flujo mayor de un orden de magnitud respecto a tramos con LW no organizados en acumulaciones estables y muy desarrolladas (diques leñosos y escalones de troncos). El valor más alto de índice de resistencia (aproximadamente 100) se registró en el caso de un tramo del torrente Tres Arroyos, en la zona austral de Chile (el número 10, cuadro 2), con mucha pendiente y caracterizado por una secuencia de escalones de troncos con pozas de socavación aguas abajo muy desarrolladas. No obstante que en la figura 4 no se tenga en cuenta la pendiente, que es una variable muy importante en la definición de la resistencia al flujo en cursos de agua de este tipo (Comiti et al. 2007), es significativo resaltar que la resistencia al flujo en tramos con acumulaciones de LW fue mayor respecto a tramos sin acumulaciones de LW.

Efecto de las acumulaciones de LW en el almacenamiento de sedimento. Sesenta y dos de las 113 acumulaciones

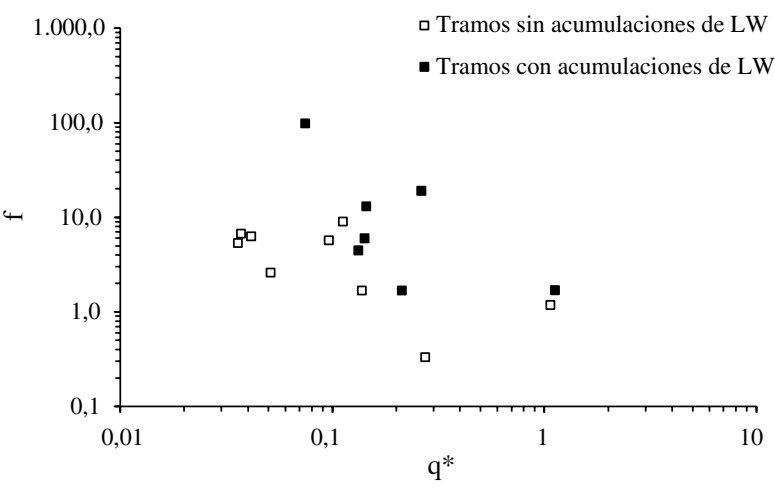

Figura 4. Relación entre la resistencia al flujo $(f)$ y el caudal unitario adimensional $\left(q^{*}\right)$ para los tramos de los torrentes Buena Esperanza y Tres Arroyos, con y sin dominancia morfológica de las acumulaciones de LW.

The Darcy-Weisbach friction factor $f$ calculated for the investigated channel reaches (with and without LW) plotted against the non-dimensional unit discharge $q^{*}$.

identificadas en el segmento del cauce estudiado del Buena Esperanza mostraron una evidente retención de sedimentos aguas arriba, especialmente en las acumulaciones de tipo transversal (diques leñosos y escalones de troncos). El volumen total geométrico del sedimento almacenado (calculado sin considerar la porosidad) fue de alrededor de $1.750 \mathrm{~m}^{3}$ $\left(959 \mathrm{~m}^{3} \mathrm{~km}^{-1}\right)$. El almacenamiento de sedimentos a escala de tramo mostró una correlación positiva con la carga de LW. El mayor coeficiente de correlación se obtuvo cuando se usó la densidad lineal numeral de detritos (piezas $\mathrm{km}^{-1}$ ) $(r=0,59 ; P<0,01)$. La figura 5 muestra la relación entre el sedimento almacenado en las acumulaciones de LW y el volumen de detrito leñoso en las acumulaciones para una submuestra $(N=46)$ de acumulaciones para las cuales se contaba con las dos mediciones. Las dos variables están significativamente correlacionadas $(r=0,54 ; P<0,01)$, aunque los diques leñosos mostraron mayor capacidad de retención de sedimento con respecto a las acumulaciones a espigón y a las acumulaciones combinadas. Esto se puede explicar por el hecho de que los diques leñosos ocupan toda la anchura del cauce y, en consecuencia, actúan como verdaderas "obras transversales orgánicas". El dique leñoso más grande (tramo 13), con un volumen de $475 \mathrm{~m}^{3}\left(H_{J},=2,3 \mathrm{~m} ; B_{J}=11 \mathrm{~m} ; L_{J}=7 \mathrm{~m}\right)$ almacenó un volumen de sedimento de aproximadamente $475 \mathrm{~m}^{3}$, disminuyendo la pendiente del cauce, incluso hasta una distancia de $40 \mathrm{~m}$ aguas arriba. El volumen de sedimentos retenido por los escalones de troncos no está reportado en la figura 5, debido a la escasa relevancia del volumen de detrito leñoso de esas acumulaciones (generalmente compuestas por un único tronco). Sin embargo, el volumen de sedimento almacenado por los escalones de troncos está, como se esperaba, altamente correlacionado con la altura del escalón $(r=0,75 ; P<0,001)$ y también con el diámetro del tronco constituyente del escalón $(r=0,63 ; P<0,01)$. 


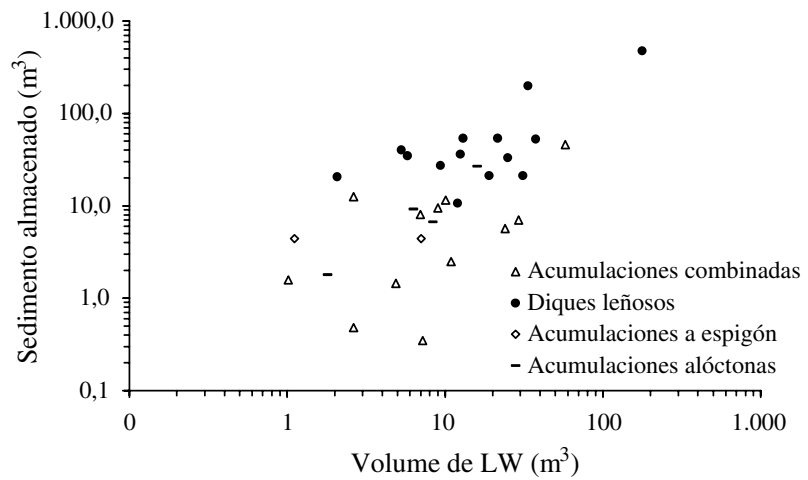

Figura 5. Volumen de material leñoso de las acumulaciones versus volumen de sedimento retenido aguas arriba. Los escalones de troncos no están reportados debido a sus bajos volúmenes leñosos.

Wood volume of jams versus the volume of upstream retained sediments. Log-steps are not plotted because of their very low LW volume.

Movimiento de los troncos marcados. En marzo de 2007 una campaña de mediciones fue dedicada a la búsqueda de los troncos marcados con etiquetas metálicas en febrero de 2006. En el periodo considerado (02/06 - 03/07) el arroyo Buena Esperanza sufrió dos avenidas $\left(2,43 \mathrm{~m}^{3} \mathrm{~s}^{-1}\right.$ el 19 de abril, y $2,40 \mathrm{~m}^{3} \mathrm{~s}^{-1}$ el 11 de noviembre de 2006) con valores de pico casi coincidentes con el caudal de cauce lleno identificado en el terreno. El caudal medio era de $0,33 \mathrm{~m}^{3} \mathrm{~s}^{-1}$. En la campaña de toma de datos, $61 \mathrm{de}$ los 380 troncos marcados no se encontraban en la posición ocupada en febrero de 2006 y puede, por tanto, considerarse que fueron transportados aguas abajo por efecto del pico de avenida más elevado $\left(2,43 \mathrm{~m}^{3} \mathrm{~s}^{-1}\right)$. Los elementos que sufrieron un transporte tenían un diámetro comprendido entre 0,1 y $0,65 \mathrm{~m}$, pero el $83 \%$ de ellos tenía un diámetro inferior a $0,25 \mathrm{~m}$ (figura 6). En lo que concierne a la longitud de los elementos transportados, el $90 \%$ de ellos tenía una longitud inferior a $3 \mathrm{~m}$ (figura 6). Cincuenta y nueve de los 61 elementos transportados se encontraban en el interior del nivel de cauce lleno, confirmando que sólo fueron móviles los elementos leñosos afectados directamente por la corriente. El $46 \%$ de los elementos transportados fue clasificado como troncos aislados y no organizados en acumulaciones. Los demás elementos eran organizados en acumulaciones dentro el cauce. De toda forma, el $81 \%$ de los elementos transportados aguas abajo estaba libre o sólo parcialmente vinculado a acumulaciones estables, grandes bloques o peñones, u otras obstrucciones en el cauce.

De los 61 elementos transportados, sólo 38 fueron encontrados aguas abajo, entendiéndose los demás como transportados más abajo del tramo considerado o en el interior del cañón donde la inspección no era practicable. De los 38 troncos individuados, el $62 \%$ estaba dispuesto en posición paralela a la corriente y no incorporado en acumulaciones. Sólo poco más del 30\% estaba acumulado con otros troncos y perpendicular a la dirección del flujo. En ningún caso un tronco recién transportado representaba un elemento de agregación para otros elementos leñosos.

\section{DISCUSIÓN}

Abundancia de troncos y características de las acumulaciones. Según estudios previos efectuados por Comiti et al. (2008) y por Andreoli (2007), la abundancia de troncos en el cauce del torrente Buena Esperanza es comparable con aquella encontrada en otros dos sitios de estudio de Los Andes chilenos de la Región de La Araucanía (río

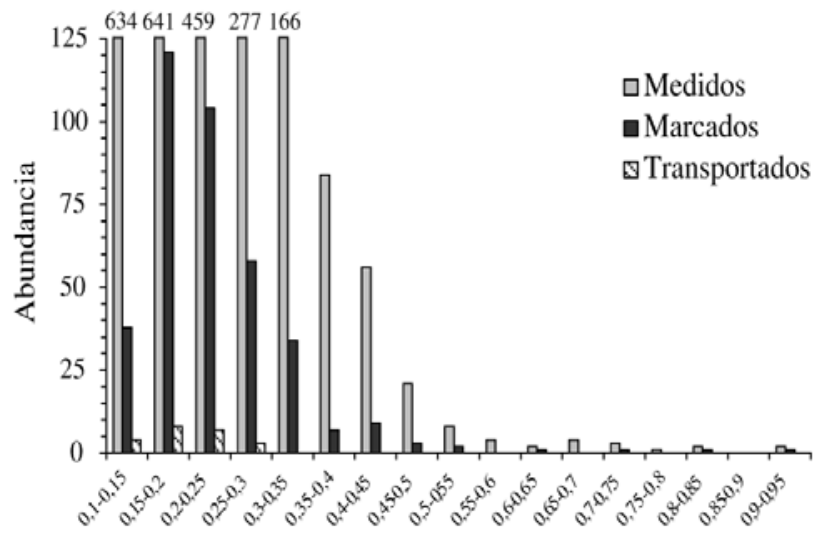

Clases de diámetros $(\mathrm{m})$

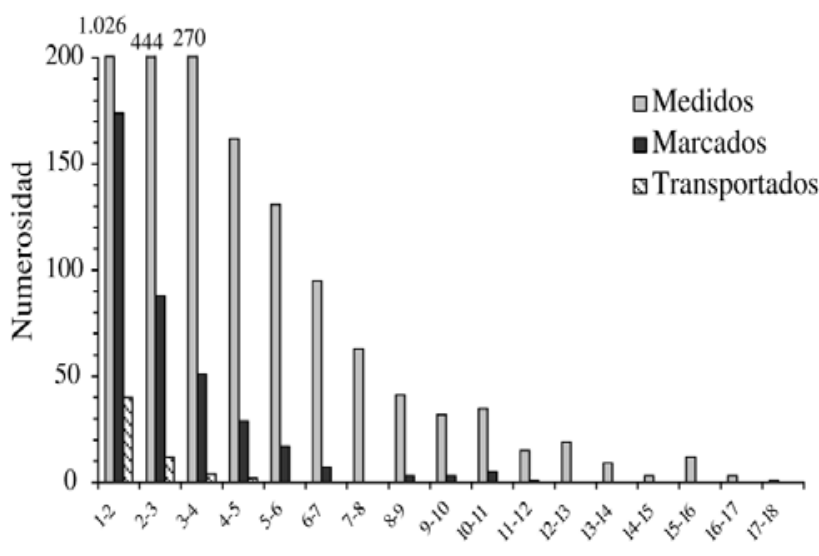

Clases de longitud $(\mathrm{m})$

Figura 6. Distribución de la abundancia en clases de diámetro y longitud: del total de los elementos medidos en el torrente Buena Esperanza, de los elementos marcados y de los elementos transportados durante la avenida con caudal máximo de $2,43 \mathrm{~m}^{3} \mathrm{~s}^{-1}$.

Frequency distribution of diameter and length size of the whole logs found in Buena Esperanza main channel, of marked logs and of logs transported by a flood of $2.43 \mathrm{~m}^{3} \mathrm{~s}^{-1}$. 
Tres Arroyos y río Toro). Al mismo tiempo, el volumen de LW del Buena Esperanza es mucho menor respecto a estos dos ríos. La causa probable de los relativamente bajos volúmenes acumulados en el torrente Buena Esperanza, junto con el pequeño diámetro de los troncos en el cauce ( $0,18 \mathrm{~m}$ en promedio), es el extremadamente bajo crecimiento de Nothofagus spp. en Tierra del Fuego (Rebertus et al. 1997, Barrera et al. 2000). Considerando también el bajo promedio de la longitud de las piezas en el cauce $(2,9 \mathrm{~m}$ versus un ancho medio de cauce lleno de $6 \mathrm{~m}$ ) no es sorprendente que el $74,7 \%$ de las piezas fuera clasificado como piezas transportadas $(66,3 \%$ considerando las piezas identificadas en todo el ancho de la planicie de inundación). No se detectaron relaciones claras entre las características del cauce y la carga de LW a escala de tramos. Esencialmente, no parecen existir correlaciones significativas $(P<0,05)$ entre el ancho $\left(B_{B F}, B_{F P}\right)$ o la pendiente del cauce $(i)$ y la densidad lineal y areal de LW. Jackson y Sturm (2002), analizando la abundancia de LW en cauces forestados de primer y segundo orden en la costa de Washington, encontraron una relación negativa entre almacenaje de LW y ancho del cauce (de 4 a $40 \mathrm{~m}$ ). Sin embargo, si sólo se consideraban ríos de primer y segundo orden en el análisis, la abundancia de LW no mostraba ninguna tendencia detectable. El rango pequeño de ancho del canal en el Buena Esperanza $(3,8 \mathrm{~m}-10,5 \mathrm{~m})$ puede ser la razón de la falta de correlación.

Tal como se describe en la literatura (Abbe y Montgomery 2003) la tipología autóctona de las acumulaciones de LW tiende a ocurrir más frecuentemente en cauces de cabecera y las acumulaciones de transporte muestran un aumento progresivo en frecuencia para ríos de mayor ancho y menor pendiente. En contraste, en el pequeño arroyo Buena Esperanza $\left(12,9 \mathrm{~km}^{2}\right)$, el número de acumulaciones en el que piezas flotantes contribuyen (acumulaciones mixtas) o constituyen una acumulación ellas mismas (acumulaciones de transporte) son predominantes $(74 \%)$.

La frecuencia espacial de 16 escalones de troncos por kilómetro lineal de cauce (cuadro 3) es comparable a los valores de 17 escalones de troncos por kilómetro encontrados en cauces de tercer orden como el Mack Creek en Oregon (Faustini y Jones 2003) y el Tres Arroyos (Comiti et al. 2008, Andreoli et al. 2007). Al mismo tiempo, los escalones de troncos son más frecuentes que lo encontrado por Marston (1982) para ríos de tercer orden en Oregon (3-4 por kilómetro) y por Comiti et al. (2006) en los Alpes de Italia (13 por kilómetro), pero menos frecuente que en la cuenca de Queets (EE.UU.) con áreas de drenaje de alrededor de $10 \mathrm{~km}^{2}$ (> 50 por kilómetro; Abbe y Montgomery 2003). Los diques leñosos en el Buena Esperanza son más frecuentes (14 por kilómetro) que los 3-4 por kilómetro encontrados por Abbe y Montgomery (2003), por Comiti et al. (2008) y por Andreoli et al. (2007). Las acumulaciones a espigón, no encontradas por Abbe y Montgomery (2003) en ríos de cuencas inferiores a
$30 \mathrm{~km}^{2}$, representan hasta un $24 \%$ de todas las acumulaciones leñosas en el torrente Buena Esperanza. Una interpretación de este patrón puede invocar las pequeñas dimensiones de los troncos del Buena Esperanza como resultado de las condiciones climáticas limitantes de la región, las que reducen el crecimiento de los árboles en los bosques de Nothofagus spp. Comparando el diámetro de las piezas medidas en el torrente Buena Esperanza con aquellas medidas en dos cuencas de tercer orden con bosque maduro (Comiti et al. 2008, Andreoli 2007) de Los Andes chilenos de la Región de La Araucanía, la mediana de los diámetros corresponde a $0,15 \mathrm{~m}$ $\left(D_{84}=0,19 \mathrm{~m}\right)$ en el Buena Esperanza, mientras que el $D_{50}$ es aproximadamente $0,30 \mathrm{~m}\left(D_{84}=0,50\right)$ en el caso de los ríos chilenos. En general, las piezas de LW en el Buena Esperanza muestran la presencia de elementos lo suficientemente grandes como para actuar como elementos clave de acumulación, junto con una gran abundancia de elementos pequeños que aumenta la frecuencia de acumulaciones combinadas y de transporte. Las acumulaciones del tipo diques leñosos y acumulaciones a espigón son las compuestas por el mayor número de piezas (cuadro 3). La acumulación con el mayor número de elementos de LW es una acumulación a espigón ubicada en el tramo 21, presentando 35 elementos, con cuatro elementos clave.

Efectos de las acumulaciones en las dimensiones e interdistancia de las pozas de socavación y en la retención de sedimentos. El volumen de las socavaciones aguas abajo de las acumulaciones de LW $(V)$ puede ser calculado a partir de las mediciones de campo de longitud $\left(L_{p}\right)$, profundidad $(s)$ y ancho $\left(B_{P}\right)$ de la poza como $V p=L_{P} * s^{*} B_{P} * 0,56$. El coeficiente de forma longitudinal $(0,56)$ deriva de experimentos de laboratorio sobre la forma de pozas de socavación aguas abajo de estructuras transversales. Los volúmenes de las pozas individuales son muy variables pero están muy correlacionados $(r=0,75 ; P<0,001)$ con la altura de las acumulaciones que las produjeron.

De las 175 pozas identificadas en el segmento estudiado del torrente Buena Esperanza, el 30\% es provocado por socavaciones aguas abajo de acumulaciones leñosas. Este valor es relativamente pequeño si es comparado con el 33-50\% observado por Evans et al. (1993) y Baillie y Davies (2002) para pequeñas cuencas con bosque nativo de Nothofagus spp. en Nueva Zelanda y con los valores observados en ríos del noroeste de la costa americana del Pacífico, de 50-75\% (Carlson et al. 1990, Montgomery et al. 1995, Beechie y Sibley 1997). Esta diferencia probablemente es debida a las pequeñas dimensiones de las piezas leñosas en el Buena Esperanza, que determinan que sólo pocas piezas sean lo suficientemente grandes como para convertirse en escalones de troncos. A escala de tramo, el porcentaje de pozas relacionadas con LW es muy variable, y no se encontraron relaciones significativas con la densidad espacial de LW. Esto confirma la poca capacidad de dar 
forma a las pozas que tienen los elementos de LW cuando no están en acumulaciones.

La distancia longitudinal entre las pozas de socavación se analiza en términos del índice de interdistancia, o sea normalizando la interdistancia entre ellas por el ancho del cauce activo de cada tramo homogéneo considerado. En el cauce en estudio, la interdistancia entre las pozas varía de siete a 0,7 veces el ancho del cauce por poza (figura 7 ). No se encontraron relaciones significativas entre este índice y la densidad espacial de $\mathrm{LW}$ (piezas $\mathrm{km}^{-1}$ ), en discordancia con estudios previos (Montgomery et al. 1995, Beechie et al. 2000) que observaron una relación inversa entre el índice de interdistancia de las pozas y la cantidad de LW en los cauces. Solamente dos tramos mostraron un índice de interdistancia mayor de tres, y ambos tramos manifiestan una morfología denominada como rápidos (cuadro 1), que no presenta pozas de socavación muy evidentes (Chin 1989). Por lo tanto, la interdistancia de las pozas está dentro del mismo rango que normalmente caracteriza los cauces con morfología de escalón-remanso sin LW en los cauces (Chin 1989). Esto parece demostrar el efecto poco significativo que la carga de LW tiene en la interdistancia entre las pozas en el Buena Esperanza. Efectivamente, la pendiente de los tramos considerados es muy alta y varía entre 0,04 y $0,09 \mathrm{~m} \mathrm{~m}^{-1}$ (excluyendo el gradiente menor del primer tramo, cuadro 1), o sea en el rango típico de cauces de escalón-remanso (Chin 1989). Considerando sólo las pozas excavadas aguas abajo de acumulaciones de detrito leñoso (escalones de troncos y diques leñosos), es evidente una relación inversa entre el índice de interdistancia de las pozas y la densidad lineal de LW (figura 7; $r=-0,63, P<0,01$ ). Estos dos resultados parecen sugerir que la contribución de detrito leñoso a la frecuencia de las pozas aumenta mientras lo hace su abundancia, pero también que este efecto es secundario en pequeños cauces de alta pendiente, donde hay un gran número de pozas relacionadas a las secuencias escalónremanso, que naturalmente caracterizan la morfología de estos cauces.

Desafortunadamente, el almacenamiento de sedimento estimado en el torrente Buena Esperanza no puede ser comparado con datos de transporte sólido de fondo. En término de parangón, para el Tres Arroyos (Los Andes chilenos, área de drenaje de $9,1 \mathrm{~km}^{2}$ ), donde hay mediciones de transporte sólido en suspensión y de fondo, Comiti et al. (2008) y Andreoli (2007) estimaron un almacenamiento de sedimentos en el cauce debido a acumulaciones de LW (diques leñosos y escalones de troncos) de $187 \%$ y $119 \%$ del transporte medio anual de fondo y total, respectivamente. Considerando el régimen hidrológico del Buena Esperanza y la escasez de surgentes activas de sedimento, se puede asumir que el transporte sólido es menor que en el torrente Tres Arroyos, con un almacenamiento de sedimento de fondo en el rango de 100\%-200\%, similar a lo reportado en cuencas de montaña de Oregon (Marston 1982).

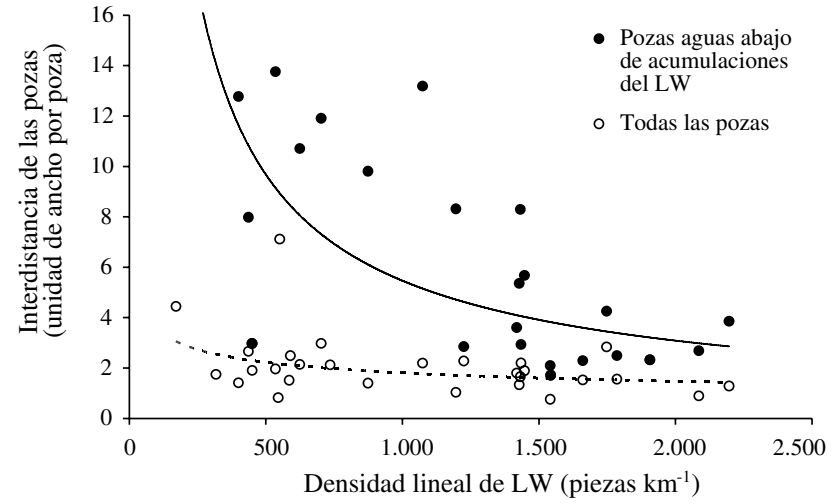

Figura 7. Densidad lineal de LW versus la interdistancia de las pozas, calculada considerando todas las pozas a lo largo de un tramo y sólo las localizadas aguas abajo de acumulaciones de LW. Las líneas representan las curvas de regresiones entre las variables.

LW linear density versus pool spacing, calculated considering all the pools along a reach and just the LW-induced ones.

Efectos de las acumulaciones de $L W$ en la resistencia al flujo. Además de la evidencia que las acumulaciones estables y bien desarrolladas de LW proporcionan un incremento de resistencia al flujo, es interesante comparar la resistencia medida en los tramos analizados con la resistencia al flujo proporcionada por la resistencia de grano. La resistencia proporcionada por la microrrugosidad (resistencia de grano) puede ser evaluada con la fórmula de Millar y Quick (1994):

$$
f_{g}=\left[2,03 \log \left(12,2 h / k_{s}\right)\right]^{-2}
$$

que relaciona la resistencia de grano $\left(f_{g}\right)$ a la sumergencia relativa $h / k_{s}$, donde $h$ es el tirante medido en campo y $k_{s}$ es la altura asignada a la rugosidad que puede ser substituida por el $D_{50}$ de la granulometría superficial (MacFarlane y Wohl 2003).

El análisis de la relación entre la resistencia de grano calculada $\left(f_{g}\right)$ y la resistencia medida en campo $(f)$, indica que la resistencia de grano es una componente que contribuye en modo menor $(<10 \%)$ a la resistencia total en tramos de ríos muy pendientes y con material detrítico organizado en estructuras como diques leñosos y escalones de troncos. En general, se confirma que los tramos de ríos de bajo orden ricos en LW, especialmente si están organizados en estructuras transversales, manifiestan granulometría más fina, estructuras de escalones más evidentes y mayor resistencia al flujo respecto a tramos sin detrito leñoso, tal como lo han reportado otros autores (MacFarlane y Wohl 2003). El aumento de resistencia al flujo puede ser atribuido más bien a la creación de macrorrugosidad, por organización de estructuras transversales y consecuente socavación de pozas aguas abajo. 
Movimiento de los troncos marcados. Las indicaciones proporcionadas por las observaciones del movimiento de troncos frente a una avenida comparable al valor de caudal de cauce lleno (recurrencia aproximada de 1-2 años) confirman que el transporte de detrito leñoso afecta sólo los elementos más pequeños y no organizados en acumulaciones estables. De hecho, en la campaña de toma de datos de campo de marzo de 2007 no fueron observadas variaciones morfológicas apreciables. Todo esto confirma cómo una avenida ordinaria no es suficiente para remodelar la estructura morfológica de un curso de agua como el considerado.

En el caso del Tres Arroyos, donde mediciones similares fueron llevadas a cabo entre 2005 y 2006, después de una avenida primaveral con pico de $4,2 \mathrm{~m}^{3} \mathrm{~s}^{-1}$ (comparable también en este caso a la de cauce lleno), sólo ocho de los 322 troncos marcados fueron transportados. La dimensión de los elementos transportados es de 0,15 - 0,45 m en diámetro y $1-5 \mathrm{~m}$ en longitud, o sea comparable a los movidos en el Buena Esperanza. También en este caso, la mayoría de los elementos transportados (62\%) no estaba organizada en acumulaciones; estaba situada en el cauce lleno, paralela a la dirección principal del flujo. La diferencia en el porcentaje de troncos transportados $(16 \%$ en el Buena Esperanza y 2,5\% en el Tres Arroyos) depende básicamente de la dimensión media de los elementos leñosos en los dos torrentes. De hecho, a causa de las diferentes condiciones climáticas entre la región templada de Chile y la subantártica de Tierra del Fuego, las medias de diámetros y longitudes de los troncos en el cauce son, respectivamente, de 0,18 y 0,5 m para el Buena Esperanza y de 0,5 y 3 m para el Tres Arroyos (Comiti et al. 2008, Andreoli 2007).

En lo que concierne al recorrido de los troncos marcados arrastrados en los torrentes Buena Esperanza y Tres Arroyos, en la figura 8 se visualiza la distancia de transporte (adimensionalizada con el diámetro del elemento) versus la relación entre la longitud del tronco y el ancho a cauce lleno. Si bien en el caso del Tres Arroyos aparece una clara correlación negativa entre los dos parámetros adimensionales, en el Buena Esperanza la dispersión de los valores es muy alta y no existe una correlación estadísticamente significativa. En este caso los datos a disposición se refieren, para los dos torrentes, a transporte debido a una avenida de recurrencia anual (condiciones de cauce lleno). El valor de estos datos se destaca porque existe una grave escasez de información de campo de transporte de material leñoso, y por la importancia de esta información para la evaluación del riesgo conectado al transporte de detritos por flotación en ríos urbanos, y para el consecuente planteamiento de las operaciones de mantención de la vegetación perifluvial en el caso de que este riesgo sea posible.

En la actualidad, el único estudio completo sobre el transporte de troncos en ríos naturales fue llevado a cabo en el Mack Creek (Oregon, USA), un torrente de tercer orden que drena una cuenca forestada por Pseudotsuga spp.

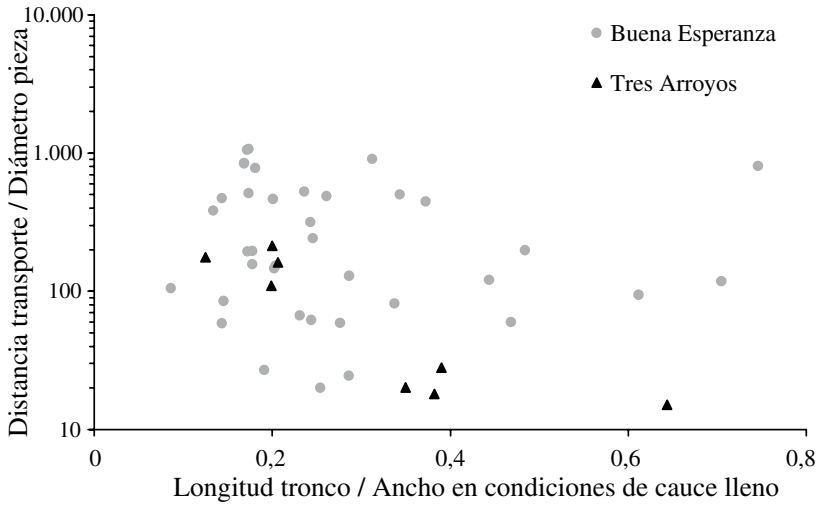

Figura 8. Relación entre la distancia de transporte de los troncos (adimensionalizada con sus diámetros) y la longitud de los troncos (adimensionalizada con el ancho en condiciones de caudal de cauce lleno).

Relationship between non-dimensional travel length and non-dimensional log length.

y Tsuga spp. Según lo reportado por Gurnell et al. (2002), ante avenidas ordinarias, menos del 1\% de los troncos está sujeto a transporte $y$, frente a una avenida con recurrencia aproximada de 25 años, esta tasa apenas supera el $10 \%$ y de éstos, muy pocos son transportados a una distancia de más de $10 \mathrm{~m}$. El mayor número de elementos movilizados por una avenida ordinaria (cauce lleno; recurrencia de 1-2 años) en el torrente Buena Esperanza fue posiblemente provocado por las dimensiones relativamente pequeñas de los elementos leñosos, y esto confirma y explica también la presencia importante de acumulaciones de tipo mixto y de transporte en el cauce del Buena Esperanza.

Influencia de la presencia de castores sobre $L W$ en otras cuencas de Tierra del Fuego. La consecuencia primaria de la presencia del Castor canadensis en los ríos del Archipiélago Fueguino es el consistente aporte de material leñoso al sistema fluvial para la construcción de diques. Un ulterior efecto indirecto es debido a la inundación del bosque de ribera, provocando la muerte en pie de los árboles que años después caen al perder capacidad de sustentación. En el primer caso, el corte de material destinado al dique corresponde a ramas y troncos de pequeño diámetro, seleccionados para su transporte. En el segundo, no habiendo selección, el material es de gran dimensión, dependiendo de las características del bosque afectado. Según Ramírez Silva (2006) en siete parcelas estudiadas en el sector chileno de Tierra del Fuego, el volumen de madera perdido en el bosque ribereño por la actividad del castor fue de $286,79 \mathrm{~m}^{3} \mathrm{ha}^{-1}$, de los cuales $280,43 \mathrm{~m}^{3} \mathrm{ha}^{-1}(97,78 \%)$ corresponden a pérdidas por inundación y $6,36 \mathrm{~m}^{3} \mathrm{ha}^{-1}(2,22 \%)$ a extracción por corte. $\mathrm{Si}$ bien estas proporciones puedan variar en función de la pendiente del terreno, más significativa en los valles de cordillera, la referencia es muy indicativa y este tipo de 
escenario es el que se manifiesta en forma dominante en las cuencas con bosque de Tierra del Fuego. Muchas de ellas, como la del Lasifashaj, en la que además de castores hay mayor porcentaje de cobertura forestal, visiblemente presentan mayor proporción de LW que en el caso del Buena Esperanza. Aunque no se pueda asumir totalmente que el Buena Esperanza representa condiciones prístinas de cuencas forestadas en Tierra del Fuego, las características de las acumulaciones de detrito leñoso y la morfología de su cauce pueden representar condiciones de referencia para la sucesiva evaluación de impactos hidrológicos, morfológicos y ecológicos determinados por las colonias de castores en otros ríos fueguinos con áreas de drenaje y pendiente del valle similares. Debido a que la magnitud del impacto de la actividad de castores en Tierra del Fuego (con excepción de la cuenca del torrente Buena Esperanza) no tiene réplica en otros lugares del mundo, no está cuantificada la significación del LW aportado por éstos en el contexto total, tema que es recomendable investigar como paso posterior a este estudio.

\section{CONCLUSIONES}

Las acumulaciones leñosas en el torrente Buena Esperanza juegan un rol importante en la geomorfología del cauce principal, pese a que el almacenamiento de grandes troncos en términos volumétricos es bajo debido a las dimensiones relativamente pequeñas de las piezas, determinado por la baja tasa de crecimiento del bosque de Nothofagus spp. en Tierra del Fuego. El tipo dominante de acumulaciones leñosas (acumulaciones mixtas) sugiere un transporte frecuente de elementos y de piezas a lo largo del cauce -probablemente causado por sus bajas dimensiones relativas- $\mathrm{y}$ tanto las acumulaciones mixtas como las de transporte muestran una frecuencia mayor que la encontrada en otros cauces de tercer orden en la costa del noroeste de Estados Unidos a lo largo de la costa del Océano Pacífico. En el Buena Esperanza, las acumulaciones leñosas son responsables de: i) creación de pozas, ii) reducción del gradiente del cauce, iii) aumento de la resistencia hidráulica y iv) almacenamiento de sedimento con valores aproximados a los $1.000 \mathrm{~m}^{3} \mathrm{~km}^{-1}$.

\section{AGRADECIMIENTOS}

Esta investigación se realizó en el ámbito de las actividades conjuntas entre las Universidades de Padova y La Plata y la Subsecretaría de Recursos Naturales de Tierra del Fuego, Argentina, previstas en el Proyecto financiado por la Unión Europea INCO-CT-2004-510735 "EPIC FORCE" (Evidence-based policy for integrated control of forested river catchments in extreme rainfall and snowmelt). Se agradece a Pablo Velásquez, José Pacheco (Subsecretaría de Recursos Naturales de Tierra del Fuego, Argentina) y Luis Opazo (Universidad Austral de Chile, Valdivia) por la ayuda y el soporte brindado en los levantamientos topográficos y en las mediciones y observaciones de campo. Un especial agradecimiento a Andrés Iroumé Arrau, profesor del Instituto de Manejo Forestal de la Universidad Austral de Valdivia, Chile, por la atenta revisión del texto.

\section{GLOSARIO}

En el texto se adoptan exclusivamente términos españoles. Para evitar posibles equivocaciones y permitir comparaciones con estudios paralelos, se reportan los términos ingleses de carácter técnico de uso común en la temática tratada y la traducción al español adoptada en el texto, ya utilizada precedentemente en otras publicaciones (Lenzi 1999, Mintegui Aguirre et al. 2006).

Bankfull discharge: caudal de cauce lleno: máxima descarga que el canal puede encauzar sin expandirse hacia su planicie de inundación (floodplain).

Boulder step: escalón formado por bloques o peñones (grandes piedras o elementos granulométricos gruesos).

Cascade: rápidos.

Combined jams: acumulaciones combinadas compuestas por elementos clave autóctonos y troncos transportados desde aguas arriba.

Floodplain: planicie de inundación.

Flow deflection jams: acumulaciones laterales, en forma de espigón o de estribos.

Large wood: troncos y fragmentos de plantas leñosas.

Large woody debris: detrito leñoso en el cauce.

Log step: escalón o salto formado por troncos dispuestos transversalmente a la corriente.

Pool: remanso o poza de socavación aguas abajo del salto.

Step: escalón o salto.

Step-pool: secuencias escalón-remanso, formadas por elementos de granulometría gruesa.

Transport jams: acumulaciones alóctonas de troncos y elementos gruesos, transportados desde aguas arriba.

Valley jams: diques leñosos que ocupan transversalmente todo el ancho del cauce y de la planicie de inundación.

\section{REFERENCIAS}

Abbe TB, DR Montgomery. 2003. Patterns and processes of wood debris accumulation in the Queets river basin, Washington. Geomorphology 51: 81-107.

Anderson NH. 1982. A survey of aquatic insects associated with wood debris in New Zealand streams. Mauri Ora 10: 21-33.

Andreoli A. 2007. Volume and Characteristics of Woody Debris in Mountain Rivers of Italian Dolomites and Southern Andes. 
Doctoral Thesis. Valdivia, Chile y Padova, Italia. Universidad Austral de Chile y Università di Padova. 116 p.

Andreoli A, G Carlig, F Comiti, A Iroumé. 2007. Estudio sobre los detritos leñosos en un río de la Cordillera de los Andes: funcionalidad e importancia. Bosque 28(2): 83-96.

Baillie BR, TR Davies. 2002. Influence of large woody debris on channel morphology in native forest and pine plantation streams in the Nelson region, New Zealand. New Zealand Journal of Marine and Freshwater Research 36: 763-774.

Barrera MD, JL Frangi, LL Richter, MH Perdomo, LB Pinedo. 2000. Structural and functional changes in Nothofagus pumilio forests along an altitudinal gradient in Tierra del Fuego, Argentina. J. Vegetation Science 11(2): 179-188.

Beechie TJ, G Pess, P Kennard, RE Bilby, S Bolton. 2000. Modeling recovery rates and pathways for woody debris recruitment in northwestern Washington streams. North American Journal of Fisheries Management 20: 436-452.

Beechie TJ, TH Sibley. 1997. Relationships between channel characteristics, woody debris, and fish habitat in northwestern Washington streams. Trans. American Fisheries Society 126: 217-229.

Bilby RE, JW Ward. 1989. Changes in characteristics and function of woody debris with increasing size of streams in western Washington. Trans. American Fisheries Society 118: 368-378.

Braudick CA, GE Grant. 2000. When do logs move in rivers? Water Resourc. Res. 36: 571-583.

Carlson JY, CW Andrus, HA Froehlich. 1990. Woody debris, channel features, and macroinvertebrates of streams with logged and undisturbed riparian timber in northeastern Oregon, U.S.A. Canadian Journal of Fisheries and Aquatic Sciences 47: 1103-1111.

Chin A. 1989. Step-pools in stream channels. Progress in Physical Geography 13(3): 391-407.

Comiti F, A Andreoli, MA Lenzi, L Mao. 2006. Spatial density and characteristics of woody debris in five mountain rivers of the Dolomites (Italian Alps). Geomorphology 78: 44-63.

Comiti F, L Mao, A Wilcox, EE Wohl, MA Lenzi. 2007. Fieldderived relationships for flow velocity and resistance in high-gradient streams. Journal of Hydrology 340: 48-62.

Comiti F, A Andreoli, L Mao, MA Lenzi. 2008. Wood storage in three mountain streams of the Southern Andes and its hydro-morphological effects. Earth Surf. Proc. Landforms 33(2): 244-262.

Cordova JM, EJ Rosi-Marshall, AM Yamamuro, GA Lamberti. 2007. Quantity, controls and functions of large woody debris in Midwestern USA streams. River Res. Applic. 23: 21-33, DOI: $10.1002 /$ rra.963.

Curran JH, EE Wohl. 2003. Large woody debris and flow resistance in step-pool channels, Cascade Range, Washington. Geomorphology 51: 141-157.

Elder K, R Kattelmann, R Ferguson. 1990. Refinements in dilution gauging for mountain streams. In Hydrology in Mountainous Regions I. IAHS Publication 193: 247-254.

Evans BF, CR Townsend, TA Crowl. 1993. Distribution and abundance of coarse woody debris in some southern New Zealand streams from contrasting forest catchments. New Zealand Journal of Marine and Freshwater Research 27: 227-239.

Faustini JM, JA Jones. 2003. Influence of large woody debris on channel morphology and dynamics in steep, boulder-rich mountain streams, western Cascades, Oregon. Geomorphology 51: $187-205$

Gurnell AM, H Piegay, SV Gregory, FJ Swanson. 2002. Large wood and fluvial processes. Freshwater Biology 47: 601-619.

Gurnell AM, R Sweet. 1998. The distribution of large woody debris accumulations and pools in relation to woodland stream management in a small, lowgradient stream. Earth Surf. Proc. Landforms 23: 1101-1121.

Ishikawa Y. 1989. Studies on disasters caused by debris flows carrying floating logs down mountain streams. Doctoral Thesis. Kyoto, Japan. Kyoto University. 121 p.

Jackson CR, CA Sturm. 2002. Woody debris and channel morphology in first- and second-order forested channels in Washington's Coast Ranges. Water Resourc. Res. 38(9): 1177, DOI: 10.1029/2001WR001138.

Keller EA, FJ Swanson. 1979. Effects of large organic material on channel form and fluvial processes. Earth Surf. Proc. Landforms 4: 361-380.

Lee AJ, RI Ferguson. 2002. Velocity and flow resistance in steppool streams. Geomorphology 46: 59-71.

Lenzi MA. 1999. Morfología y estabilidad de las secuencias en escalones en los torrentes alpinos de elevada pendiente. Ingeniería del Agua 6: 151-162.

Lienkaemper GW, FJ Swanson. 1987. Dynamics of large woody debris in streams in old-growth Douglas-fir forests. Can. J. For. Res. 17: 150-156.

Lizarralde M, J Escobar, G Deferrari. 2004. Invader species in Argentina: A review about the beaver (Castor Canadensis) population situation on Tierra del Fuego ecosystem. Interciencia 29: 352-356.

MacFarlane WA, EE Wohl. 2003. Influence of step composition on step geometry and flow resistance in step-pool streams of the Washington Cascades. Water Resourc. Res. 39(2): W1037, DOI: 10.1029/2001WR001238.

Mao L, GP Uyttendaele, A Iroumé, MA Lenzi. 2008. Field based analysis of sediment entrainment in two high gradient streams located in Alpine and Andine environments. Geomorphology 93: 368-383.

Marston RA. 1982. The geomorphic significance of log steps in forest streams. Annals of the Association of American Geographers 72(1): 99-108.

Millar RG, MC Quick. 1994. Flow resistance of high-gradient gravel channels. In Cotroneo GV, RR Rumer eds. Hydraulic Engineering '94. ASCE. p. 717-721.

Mintegui Aguirre JA, MA Lenzi, JC Robredo Sánchez, L Mao. 2006. Movilización versus estabilización de los sedimentos en los cursos sometidos a la dinámica torrencial. Madrid, España. Organismo Autónomo Parques Nacionales, Ministerio de Medio Ambiente. 143 p.

Montgomery DR, JM Buffington, RD Smith, KM Schmidt, G Pess. 1995. Pool spacing in forest channels. Water Resourc. Res. 31(4): 1097-1105.

Montgomery DR, JM Buffington. 1997. Channel-reach morphology in mountain drainage basins. Geological Society of America Bulletin 109: 596-611.

Nakamura F, FJ Swanson. 1993. Effects of coarse woody debris on morphology and sediment storage of a mountain stream system in western Oregon. Earth Surf. Proc. Landforms 18: 43-61.

Rebertus AJ, T Kitzberger, TT Veblen, LM Roovers. 1997. Blowdown history and landscape patterns in the Andes of Tierra del Fuego, Argentina. Ecology 78(3): 678-692. 
Ramírez Silva MD. 2006. Cuantificación de la biomasa leñosa removida por Castor canadensis (Kuhl 1820, Rodentia) en bosques nativos de Tierra del Fuego (XII Región de Magallanes, Chile). Tesis de grado. Pontificia Universidad Católica de Chile. 151 p.

Shields FD, CJ Gippel. 1995. Prediction of effects of woody debris removal on flow resistance. Journal of Hydraulic Engineering 121: 341-354.

Smith RD, RC Sidle, PE Porter. 1993. Effects of bedload transport of experimental removal of woody debris from a forest gravel-bed stream. Earth Surf. Proc. Landforms 18: 455-468.

Strahler AN. 1952. Dynamic basis of geomorphology. Geological Society of America Bulletin 63: 923-938.

Swanson FJ, GW Lienkaemper. 1984. Interactions among fluvial processes, forest vegetation, and aquatic ecosystems, South Fork Hoh River, Olympic National Park. In Starkey EE, JF Franklin, JW Matthews eds. Proc. $2^{\text {nd }}$ Conference on Scientific Research in the National Parks, Oregon State Univ., Corvallis, OR. p. 30-34.

Swanson FJ, RC Fredriksen. 1982. Sediment routing and budget implications for judging impacts of forestry practices. In Swanson FJ, RJ Janda, T Dunne, DN Swanston eds. Sediment Budgets and Routing in Forested Drainage Basins. USDA Forest Service General Technical Report 141. p. 129-137.

Thevenet A, A Citterio, H Piegay. 1998. A new methodology for the assessment of large woody debris accumulations on highly modified rivers (example of two French Piedmont rivers). Regulated Rivers: Research \& Management 14(6): 467-483.

Thompson DM. 1995. The effects of large organic debris on sediment processes and stream morphology in Vermont. Geomorphology 11: 234-235.

Uyttendaele GP. 2006. Procesos de transporte de sedimentos en áreas de montaña, comparación entre la cuenca del Río Cordón (Alpes, Italia) y el Estero los Tres Arroyos (Andes, Chile). Thesis Doctoral. Valdivia, Chile y Padova, Italia. Universidad Austral de Chile y Università di Padova. 236 p.

Wilcox A, JM Nelson, EE Wohl. 2006. Flow resistance dynamics in step-pool channels: 2. Partitioning between grain, spill, and woody debris resistance. Water Resourc. Res. 42, W05419, DOI:10.1029/2005WR00427.

Wohl EE, A Wilcox. 2005. Channel geometry of mountain streams in New Zealand. J. Hydrology 300: 252-266. doi:10.1016/j. jhydrol.2004.06.006.

Wohl E, S Madsen, L MacDonald. 1997. Characteristics of log and clast bed-steps in step-pool streams of northwestern Montana, USA. Geomorphology 20: 1-10.

Wood PJ, AP Dykes. 2002. The use of salt dilution gauging techniques: ecological considerations and insights. Water Research 36: 3054-3062.

Young MK. 1994. Movement and characteristics of stream-bourne coarse woody debris in adjacent burned and undisturbed watersheds in Wyoming. Can. J. For. Res. 24: 1933-1938. 